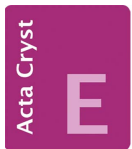

CRYSTALLOGRAPHIC COMMUNICATIONS

ISSN 2056-9890

Received 17 February 2020

Accepted 14 March 2020

Edited by A. V. Yatsenko, Moscow State University, Russia

Keywords: crystal structure; acesulfame; 2 amino-3-hydroxypyridine; hydrogen bonding; Hirshfeld surface analysis.

CCDC reference: 1920140

Supporting information: this article has supporting information at journals.iucr.org/e
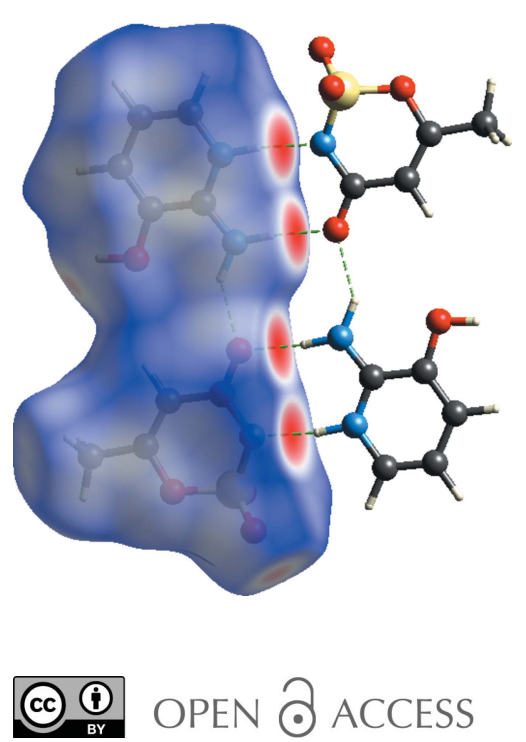

\section{Crystal structure and Hirshfeld surface analysis of 2-amino-3-hydroxypyridin-1-ium 6-methyl-2,2,4- trioxo-2H,4H-1,2,3-oxathiazin-3-ide}

\author{
Sevgi Kansiz, ${ }^{\text {a* }}$ Md. Serajul Haque Faizi, ${ }^{\mathrm{b}}$ Tansu Merve Aydin, ${ }^{\mathrm{c}}$ Necmi Dege, ${ }^{\mathrm{d}}$ \\ Hasan Icbudak ${ }^{c}$ and Irina A. Golenya ${ }^{e^{*}}$
}

aDepartment of Fundamental Sciences, Faculty of Engineering, Samsun University, Samsun, 55420, Turkey, bPG
Department of Chemistry, Langat Singh College, B. R. A. Bihar University, Muzaffarpur, Bihar-842001, India,
cDepartment of Chemistry, Faculty of Arts and Sciences, Ondokuz Mayıs, University, Samsun, 55200, Turkey,
${ }^{\text {d} D e p a r t m e n t ~ o f ~ P h y s i c s, ~ F a c u l t y ~ o f ~ A r t s ~ a n d ~ S c i e n c e s, ~ O n d o k u z ~ M a y ı s, ~ U n i v e r s i t y, ~ S a m s u n, ~ 55200, ~ T u r k e y, ~ a n d ~}$
e Department of Chemistry, Volodymyrska str., 64, National Taras Shevchenko University, 01601 Kyiv, Ukraine.
${ }^{*}$ Correspondence e-mail: sevgi.kansiz85@gmail.com, igolenya@ua.fm

The asymmetric unit of the title compound, $\mathrm{C}_{5} \mathrm{H}_{7} \mathrm{~N}_{2} \mathrm{O}^{+} \cdot \mathrm{C}_{4} \mathrm{H}_{4} \mathrm{NO}_{4} \mathrm{~S}^{-}$, contains one cation and one anion. The 6-methyl-2,2,4-trioxo- $2 \mathrm{H}, 4 \mathrm{H}$-1,2,3-oxathiazin-3ide anion adopts an envelope conformation with the $\mathrm{S}$ atom as the flap. In the crystal, the anions and cations are held together by $\mathrm{N}-\mathrm{H} \cdots \mathrm{O}, \mathrm{N}-\mathrm{H} \cdots \mathrm{N}, \mathrm{O}-$ $\mathrm{H} \cdots \mathrm{O}$ and $\mathrm{C}-\mathrm{H} \cdots \mathrm{O}$ hydrogen bonds, thus forming a three-dimensional structure. The Hirshfeld surface analysis and fingerprint plots reveal that the crystal packing is dominated by $\mathrm{O} \cdots \mathrm{H} / \mathrm{H} \cdots \mathrm{O}(43.1 \%)$ and $\mathrm{H} \cdots \mathrm{H}(24.2 \%)$ contacts.

\section{Chemical context}

Food additives are substances added intentionally to foodstuffs to perform certain functions such as to impart colour, to sweeten or preserve. They play an essential role in the modern food industry, supporting quality and safety. In this context, artificial sweeteners are widely used in food, beverage, confectionery and pharmaceutical products throughout the world (Clauss \& Jensen, 1973; Ni et al., 2009). Oxathiazinone dioxide, systematic name 6-methyl-1,2,3-oxathiazin- 4(3H)one 2,2-dioxide and also known as 6-methyl-3,4-dihydro-1,2,3oxathiazin-4-one 2,2-dioxide or acesulfame, has been widely used as a non-caloric artificial sweetener (Duffy \& Anderson, 1998) since 1988, after the FDA (US Food and Drug Administration) granted approval. Many countries have approved the use of acesulfame- $\mathrm{K}$ in soft drinks, toothpaste, candies, mouthwash, cosmetics and pharmacological preparations (Mukherjee \& Chakrabarti, 1997). The chemistry of acesulfame is of interest not only because of its biological importance but also in relation to its coordination properties, since the acesulfame anion offers different donor atoms to metal ions, namely the imino nitrogen, ring oxygen, one carbonyl and two sulfonyl oxygen atoms. To advance the knowledge of such compounds, we report the synthesis, single crystal structure determination and Hirshfeld surface analysis of the 2-amino-3-hydroxypyridinium acesulfamate salt (I).

\section{Structural commentary}

A view of the asymmetric unit of (I) with the atom-numbering scheme is shown in Fig. 1. In the acesulfamate anion, the bond 
Table 1

Selected bond lengths $(\AA)$.

\begin{tabular}{llll}
\hline $\mathrm{S} 1-\mathrm{O} 2$ & $1.4149(17)$ & $\mathrm{O} 5-\mathrm{C} 6$ & $1.353(3)$ \\
$\mathrm{S} 1-\mathrm{O} 3$ & $1.4235(18)$ & $\mathrm{O} 4-\mathrm{C} 1$ & $1.236(3)$ \\
$\mathrm{S} 1-\mathrm{N} 1$ & $1.5605(17)$ & $\mathrm{N} 2-\mathrm{C} 5$ & $1.336(3)$ \\
$\mathrm{S} 1-\mathrm{O} 1$ & $1.6204(15)$ & $\mathrm{N} 3-\mathrm{C} 5$ & $1.317(3)$ \\
$\mathrm{O} 1-\mathrm{C} 3$ & $1.383(2)$ & $\mathrm{N} 1-\mathrm{C} 1$ & $1.359(3)$ \\
\hline
\end{tabular}

dimensions correspond to the given structural formula with double bonds $\mathrm{C} 1=\mathrm{O} 4$ and $\mathrm{C} 2=\mathrm{C} 3$ and a single bond $\mathrm{C} 1-\mathrm{C} 2$ (Table 1). A relatively short $\mathrm{N} 1-\mathrm{C} 1$ bond indicates strong $\pi$ conjugation in the $\mathrm{N} 1-\mathrm{C} 1=\mathrm{O} 4$ fragment. Overall, the bond lengths in this anion compare well with those observed in other acesulfamate salts known from the literature (Yıldirım et al., 2019; Kansiz et al., 2019). The six-membered acesulfamate ring adopts an envelope conformation with atom S1 as the flap; its deviation from the basal plane is 0.555 (1) $\AA$. The basal plane of the envelope is slightly twisted, with an $\mathrm{O} 1-$ $\mathrm{C} 3-\mathrm{C} 1-\mathrm{N} 1$ torsion angle of $2.2(2)^{\circ}$. The cyclic bond lengths in the 2-amino-3-hydroxypyridinium cation agree well with its aromatic nature. The short N3-C5 distance indicates strong conjugation of the amino $\mathrm{N} 3$ atom with the acceptor $\pi$-system of the pyridinium ring. The cation is almost planar, the largest deviation from the least-squares plane of 0.008 (2) $\AA$ is observed for atom C6. The least-squares planes through the cation and the basal atoms of anion form a dihedral angle of $6.47(11)^{\circ}$.<smiles></smiles>

\section{Supramolecular features}

The acesulfamate anions are linked to the 2-amino-3-hydroxypyridinium cations by strong $\mathrm{N}-\mathrm{H} \cdots \mathrm{N}$ and $\mathrm{N}-\mathrm{H} \cdots \mathrm{O}$

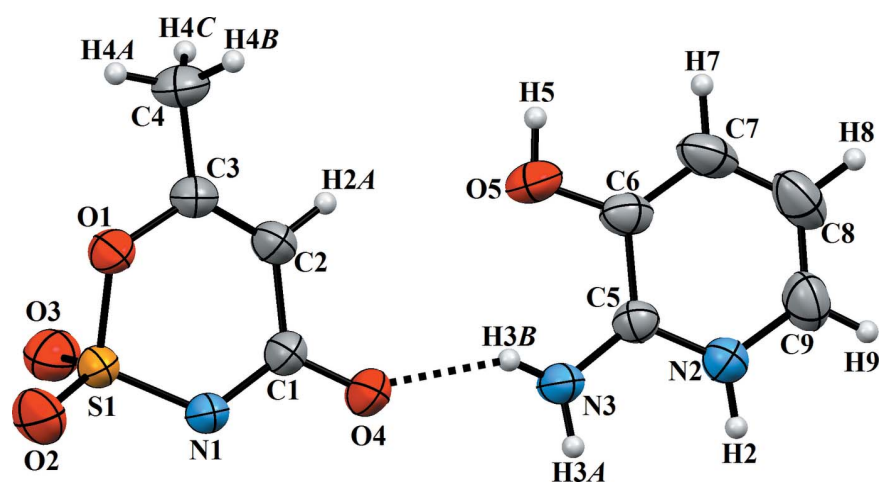

Figure 1

A view of the asymmetric unit of the title compound with the atomnumbering scheme. Displacement ellipsoids are drawn at the $50 \%$ probability level. The $\mathrm{N}-\mathrm{H} \cdots \mathrm{O}$ hydrogen bond is shown as a dashed line.
Table 2

Hydrogen-bond geometry $\left(\AA,^{\circ}\right)$.

\begin{tabular}{lllll}
\hline$D-\mathrm{H} \cdots A$ & $D-\mathrm{H}$ & $\mathrm{H} \cdots A$ & $D \cdots A$ & $D-\mathrm{H} \cdots A$ \\
\hline $\mathrm{N} 3-\mathrm{H} 3 B \cdots \mathrm{O} 4$ & $0.81(3)$ & $2.10(3)$ & $2.808(3)$ & $145(2)$ \\
$\mathrm{N} 3-\mathrm{H} 3 A \cdots \mathrm{O} 4^{\mathrm{i}}$ & $0.85(3)$ & $2.00(3)$ & $2.846(3)$ & $175(3)$ \\
$\mathrm{N}^{\mathrm{H}}-\mathrm{H} 2 \cdots \mathrm{N}^{\mathrm{i}}$ & $0.90(3)$ & $1.99(3)$ & $2.871(3)$ & $168(3)$ \\
$\mathrm{O}^{\mathrm{ii}}-\mathrm{H} 5 \cdots 3^{3 i}$ & $0.84(4)$ & $2.50(4)$ & $3.090(2)$ & $128(3)$ \\
$\mathrm{O}^{\mathrm{O}}-\mathrm{H} 5 \cdots \mathrm{O} 3^{\text {iii }}$ & $0.84(4)$ & $2.25(4)$ & $2.995(2)$ & $147(3)$ \\
$\mathrm{C} 8-\mathrm{H} 8 \cdots \mathrm{O} 2^{\text {iv }}$ & 0.93 & 2.49 & $3.402(3)$ & 166 \\
\hline
\end{tabular}

hydrogen bonds, forming centrosymmetric aggregates each consisting of two cations and two anions (Table 2, Fig. 2).

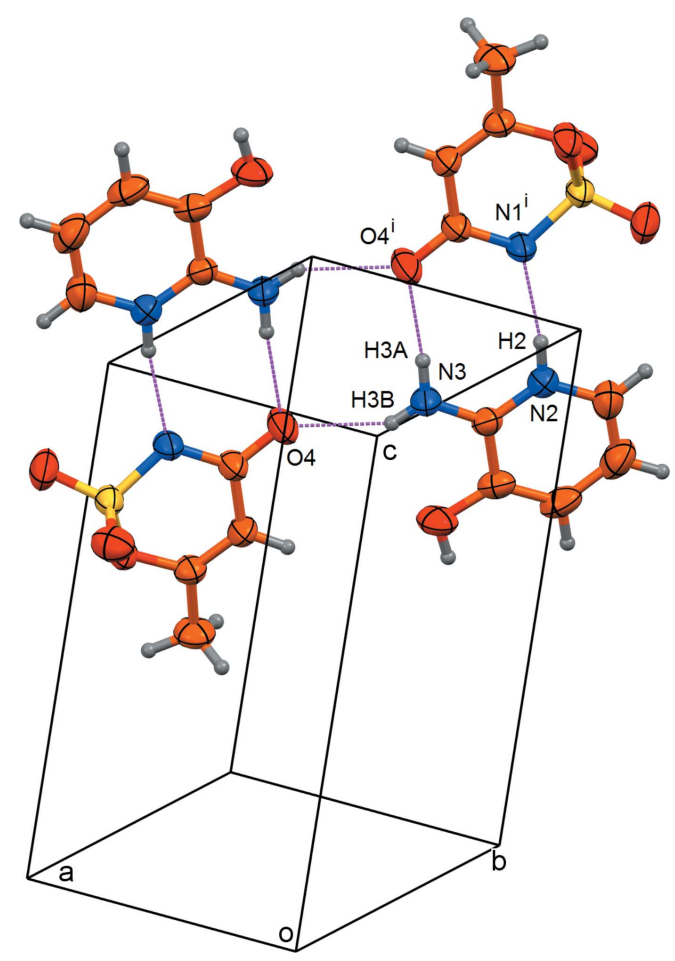

Figure 2

A view of the centrosymmetric aggregate formed by strong $\mathrm{N}-\mathrm{H} \cdots \mathrm{N}$ and $\mathrm{N}-\mathrm{H} \cdots \mathrm{O}$ hydrogen bonds (dashed lines). Displacement ellipsoids are drawn at the $50 \%$ probability level. Symmetry code: (i) $1-x, 1-y$, $2-z$.

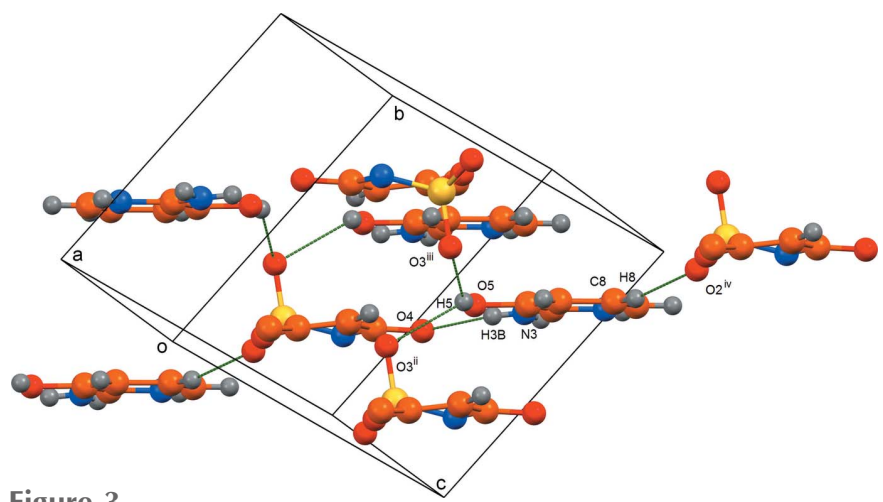

Figure 3

A view of the crystal packing of the title compound showing the threedimensional system of hydrogen bonds. Methyl $\mathrm{H}$ atoms are omitted for clarity. Symmetry codes: (ii) $x-1, y, z$; (iii) $1-x, 1-y, 1-z$; (iv) $x-2$, $y+1, z$. 
These aggregates are linked into a three-dimensional structure by weak $\mathrm{O}-\mathrm{H} \cdots \mathrm{O}$ hydrogen bonds involving the sulfonyl groups and by $\mathrm{C}-\mathrm{H} \cdots \mathrm{O}$ contacts (Table 2, Fig. 3). The shortest intercentroid separation in (I) is only 4.1798 (15) $\AA$, and thus the $\pi$-stacking interactions in this structure are insignificant.

\section{Database survey}

A search of the Cambridge Structural Database (CSD, version 5.39; Groom et al., 2016) gave 54 hits for the oxathiazin moiety. The compounds most closely related to (I) are 3-carbamoylpyridin-1-ium 6-methyl-2,2,4-trioxo- $2 \mathrm{H}, 4 \mathrm{H}$-1,2,3-oxathiazin-3-ide hemihydrate (CIHDEF; Wang et al., 2018), 3carboxypyridin-1-ium 6-methyl-2,2,4-trioxo- $2 \mathrm{H}, 4 \mathrm{H}$-1,2,3-oxathiazin-3-ide (CIHDIJ; Wang et al., 2018), 6-amino-2-oxo-2,3dihydropyrimidin-1-ium 6-methyl-2,2,4-trioxo-2 $H, 4 H-1,2,3-$ oxathiazin-3-ide 4-aminopyrimidin-2(1H)-one (CIHFEH; Wang et al., 2018), 5-fluoro-2-oxo-2,3-dihydropyrimidin4(1H)-iminium 6-methyl-4-oxo-4H-1,2,3-oxathiazin-3-ide 2,2dioxide hemihydrate (GONLIG; Wang et al., 2014), potassium 6-methyl-1,2,3-oxathiazin-4-one-2,2-dioxide (KMOTZD; Paulus 1975), thallium(I) 6-methyl-4-oxo-4H-1,2,3-oxathiazin3-ide 2,2-dioxide (OCAHUY; Baran et al., 2015), choline acesulfamate (ODIHOZ; Nockemann et al., 2007) and rubidium 6-methyl-4-oxo-4H-1,2,3-oxathiazin-3-ide 2,2dioxide (SURCIT; Piro et al., 2015). In GONLIG, the molecules are linked by $\mathrm{N}-\mathrm{H} \cdots \mathrm{O}$ hydrogen bonds, as in the title compound. In SURCIT, the carbonyl $\mathrm{C}=\mathrm{O}$ bond distance is

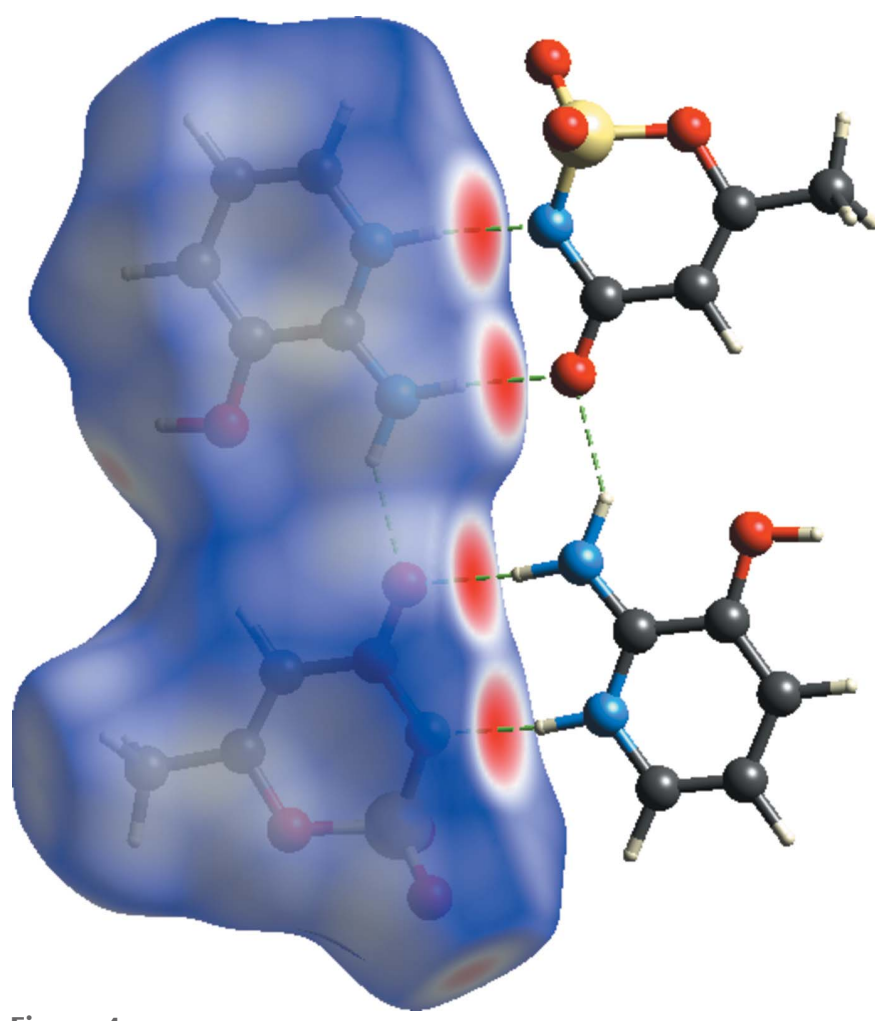

Figure 4

A view of the three-dimensional Hirshfeld surface of the title compound plotted over $d_{\text {norm }}$ in the range -0.5966 to +1.0568 a.u.
1.231 (5) $\AA$ and the sulfoxide $\mathrm{S}=\mathrm{O}$ bond lengthsare 1.415 (3) and 1.421 (3) $\AA$, which are close toose in the title compound.

\section{Hirshfeld surface analysis}

In order to visualize the intermolecular interactions in the crystal of (I), Hirshfeld surface analysis (Spackman \& Jayatilaka, 2009) was carried out using CrystalExplorer17.5 (Turner et al., 2017). Fig. 4 shows the Hirshfeld surface and the intermolecular contacts of the title compound mapped over $d_{\text {norm }}$ in the range -0.5966 to +1.0568 a.u. The red regions (distances shorter than the sum of the van der Waals radii) are apparent around the oxygen atom $\mathrm{O} 4$, which participates in the $\mathrm{N}-$ $\mathrm{H} \cdots \mathrm{O}$ contacts, and around the nitrogen atom $\mathrm{N} 1$, which participates in the $\mathrm{N}-\mathrm{H} \cdots \mathrm{N}$ contacts (Fig. 2, Table 2). The fingerprint plots for (I) are given in Fig. 5. The largest contribution to the overall crystal packing is from $\mathrm{O} \cdots \mathrm{H} /$ $\mathrm{H} \cdots \mathrm{O}$ interactions $(43.1 \%) . \mathrm{H} \cdot \mathrm{H}$ contacts provide another significant contribution to the Hirshfeld surface of $24.2 \%$. The
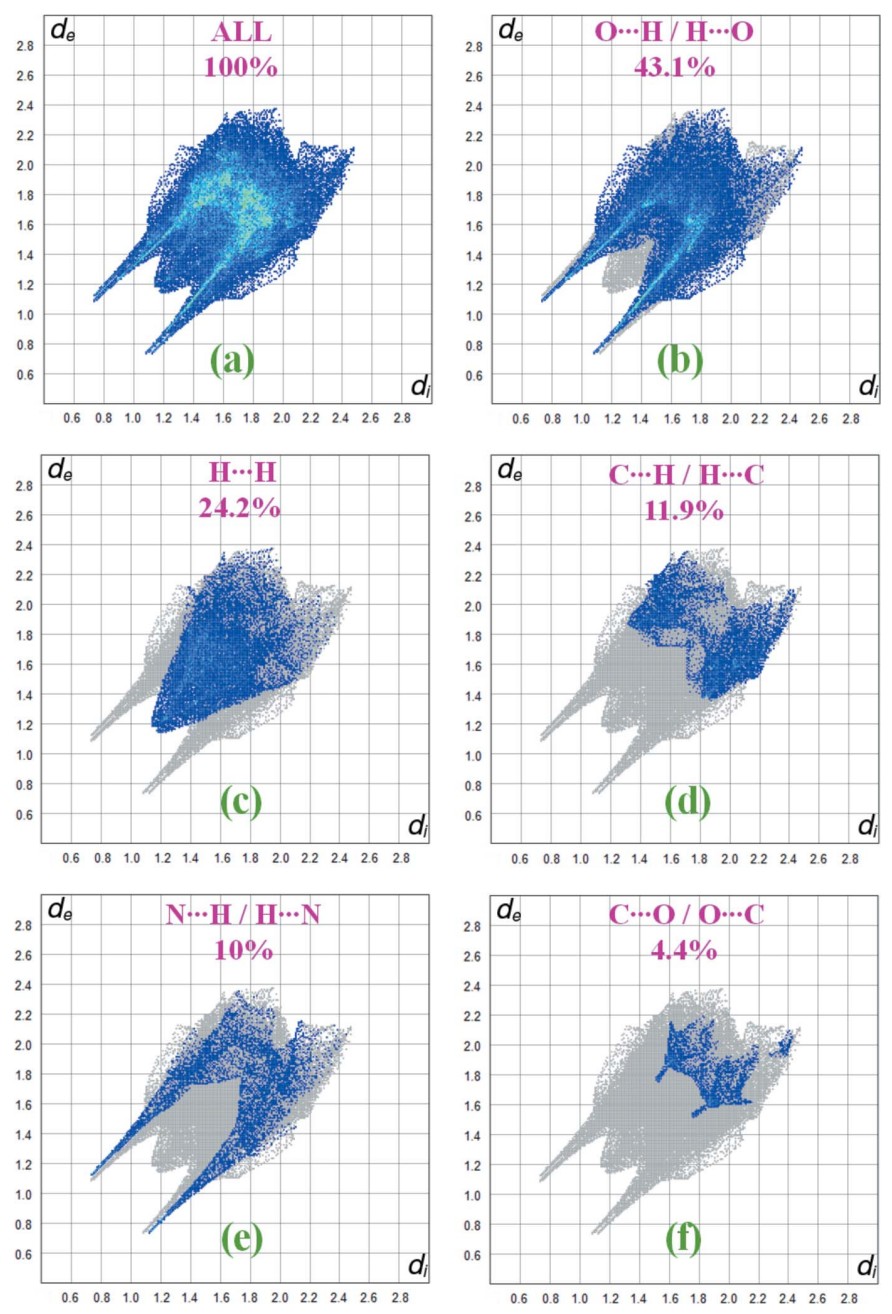

Figure 5

The two-dimensional fingerprint plots for the title compound showing $(a)$ all interactions, (b) $\mathrm{O} \cdots \mathrm{H} / \mathrm{H} \cdots \mathrm{O},(c) \mathrm{H} \cdots \mathrm{H},(d) \mathrm{C} \cdots \mathrm{H} / \mathrm{H} \cdots \mathrm{C},(e)$ $\mathrm{N} \cdots \mathrm{H} / \mathrm{H} \cdots \mathrm{N}$ and $(f) \mathrm{C} \cdots \mathrm{O} / \mathrm{O} \cdots \mathrm{C}$ interactions. 
Table 3

Experimental details.

\begin{tabular}{|c|c|}
\hline \multicolumn{2}{|l|}{ Crystal data } \\
\hline Chemical formula & $\mathrm{C}_{5} \mathrm{H}_{7} \mathrm{~N}_{2} \mathrm{O}^{+} \cdot \mathrm{C}_{4} \mathrm{H}_{4} \mathrm{NO}_{4} \mathrm{~S}^{-}$ \\
\hline$M_{\mathrm{r}}$ & 273.27 \\
\hline Crystal system, space group & Triclinic, $P \overline{1}$ \\
\hline Temperature (K) & 296 \\
\hline$a, b, c(\AA)$ & 7.1676 (5), 9.1175 (7), 10.1554 (8) \\
\hline$\alpha, \beta, \gamma\left({ }^{\circ}\right)$ & $66.174(6), 80.225(6), 71.803(6)$ \\
\hline$V\left(\AA^{3}\right)$ & $576.01(8)$ \\
\hline$Z$ & 2 \\
\hline Radiation type & Мо $K \alpha$ \\
\hline$\mu\left(\mathrm{mm}^{-1}\right)$ & 0.30 \\
\hline Crystal size $(\mathrm{mm})$ & $0.57 \times 0.42 \times 0.21$ \\
\hline \multicolumn{2}{|l|}{ Data collection } \\
\hline Diffractometer & STOE IPDS 2 \\
\hline Absorption correction & $\begin{array}{l}\text { Integration ( } X \text {-RED32; Stoe \& Cie, } \\
\text { 2002) }\end{array}$ \\
\hline$T_{\min }, T_{\max }$ & $0.855,0.953$ \\
\hline $\begin{array}{l}\text { No. of measured, independent and } \\
\text { observed }[I>2 \sigma(I)] \text { reflections }\end{array}$ & $5116,2263,1951$ \\
\hline$R_{\text {int }}$ & 0.031 \\
\hline$(\sin \theta / \lambda)_{\max }\left(\AA^{-1}\right)$ & 0.617 \\
\hline \multicolumn{2}{|l|}{ Refinement } \\
\hline$R\left[F^{2}>2 \sigma\left(F^{2}\right)\right], w R\left(F^{2}\right), S$ & $0.040,0.114,1.08$ \\
\hline No. of reflections & 2263 \\
\hline No. of parameters & 179 \\
\hline $\mathrm{H}$-atom treatment & $\begin{array}{l}\mathrm{H} \text { atoms treated by a mixture of } \\
\text { independent and constrained } \\
\text { refinement }\end{array}$ \\
\hline$\Delta \rho_{\max }, \Delta \rho_{\min }\left(\mathrm{e} \AA^{-3}\right)$ & $0.34,-0.26$ \\
\hline
\end{tabular}

Computer programs: $X$-AREA and X-RED (Stoe \& Cie, 2002), SHELXT2017 (Sheldrick, 2015a), SHELXL2017 (Sheldrick, 2015b), PLATON (Spek, 2020) and Win $G X$ (Farrugia, 2012).

$\mathrm{N} \cdots \mathrm{H} / \mathrm{H} \cdots \mathrm{N}$ contacts appear as a pair of characteristic tips in the fingerprint plots; they contribute $10 \%$ to the Hirshfeld surface (Table 2).

\section{Synthesis and crystallization}

Potassium acesulfamate $(1 \mathrm{mmol})$ was dissolved in $15 \mathrm{~mL}$ ethanol and heated to $348 \mathrm{~K}$. To this solution $1 \mathrm{mmol}$ of 2 amino-3-hydroxypyridine in $15 \mathrm{~mL}$ of ethanol was added slowly under continuous stirring. After the addition, the solution was stirred for another $6 \mathrm{~min}$ at the same temperature. The compound thus formed was separated from the solution and then recrystallized from ethanol solution at room temperature. The red needle-shaped crystals obtained were filtered, washed with ethyl acetate and dried, yield $91 \%$.

\section{Refinement}

Crystal data, data collection and structure refinement details are summarized in Table 3. All C-bound hydrogen atoms were placed in idealized positions and refined isotropically using a riding model, with $U_{\text {iso }}(\mathrm{H})=1.5 U_{\text {eq }}(\mathrm{C})$ for methyl and with $U_{\text {iso }}(\mathrm{H})=1.2 U_{\text {eq }}(\mathrm{C})$ for other $\mathrm{C}$ atoms, $\mathrm{C}-\mathrm{H}=0.96 \AA$ for methyl and $0.93 \AA$ for $s p^{2}$-hybridized $\mathrm{C}$ atoms. All other $\mathrm{H}$ atoms were located from the difference map and refined freely.

\section{Funding information}

This study was supported by Ondokuz Mayıs University under project No. PYO·FEN.1906.19.001.

\section{References}

Baran, E. J., Parajón-Costa, B. S., Echeverría, G. A. \& Piro, O. E. (2015). Maced. J. Chem. Chem. Eng. 34, 95-100.

Clauss, K. \& Jensen, H. (1973). Angew. Chem. Int. Ed. Engl. 12, 869876.

Duffy, V. D. \& Anderson, G. H. (1998). J. Am. Diet. Assoc. 98, 580587.

Farrugia, L. J. (2012). J. Appl. Cryst. 45, 849-854.

Groom, C. R., Bruno, I. J., Lightfoot, M. P. \& Ward, S. C. (2016). Acta Cryst. B72, 171-179.

Kansız, S., Tolan, A., İçbudak, H. \& Dege, N. (2019). J. Mol. Struct. 1190, 102-115.

Mukherjee, A. \& Chakrabarti, J. (1997). Food Chem. Toxicol. 35, $1177-1179$.

Ni, Y., Xiao, W. \& Kokot, S. (2009). Food Chem. 113, 1339-1345.

Nockemann, P., Thijs, B., Driesen, K., Janssen, C. R., Van Hecke, K., Van Meervelt, L., Kossmann, S., Kirchner, B. \& Binnemans, K. (2007). J. Phys. Chem. B, 111, 5254-5263.

Paulus, E. F. (1975). Acta Cryst. B31, 1191-1193.

Piro, O. E., Echeverría, G. A., Castellano, E. E., Parajón-Costa, B. S. \& Baran, E. J. (2015). Z. Naturforsch. Teil B, 70, 491-496.

Sheldrick, G. M. (2015a). Acta Cryst. A71, 3-8.

Sheldrick, G. M. (2015b). Acta Cryst. C71, 3-8.

Spackman, M. A. \& Jayatilaka, D. (2009). CrystEngComm, 11, 19-32.

Spek, A. L. (2020). Acta Cryst. E76, 1-11.

Stoe \& Cie (2002). $X$-AREA and $X$-RED32. Stoe \& Cie $\mathrm{GmbH}$, Darmstadt, Germany.

Turner, M. J., MacKinnon, J. J., Wolff, S. K., Grimwood, D. J., Spackman, P. R., Jayatilaka, D. \& Spackman, M. A. (2017). Crystal Explorer17.5. University of Western Australia. http://hirshfeldsurface.net.

Wang, C., Perumalla, S. R. \& Sun, C. C. (2018). Cryst. Growth Des. 18, 4215-4219.

Wang, L., Wen, X., Li, P., Wang, J., Yang, P., Zhang, H. \& Deng, Z. (2014). CrystEngComm, 16, 8537-8545.

Yıldırım, T., Köse, D. A., Avcı, E., Özer, D. \& Şahin, O. (2019). J. Mol. Struct. 1176, 576-582. 


\section{supporting information}

Acta Cryst. (2020). E76, 572-575 [https://doi.org/10.1107/S2056989020003813]

Crystal structure and Hirshfeld surface analysis of 2-amino-3-hydroxypyridin-1ium 6-methyl-2,2,4-trioxo-2H,4H-1,2,3-oxathiazin-3-ide

Sevgi Kansiz, Md. Serajul Haque Faizi, Tansu Merve Aydin, Necmi Dege, Hasan Icbudak and

Irina A. Golenya

Computing details

Data collection: STOE $X$-AREA (Stoe \& Cie, 2002); cell refinement: STOE $X$-AREA (Stoe \& Cie, 2002); data reduction: STOE X-RED (Stoe \& Cie, 2002); program(s) used to solve structure: SHELXT2017 (Sheldrick, 2015a); program(s) used to refine structure: SHELXL2017 (Sheldrick, 2015b); molecular graphics: PLATON (Spek, 2020); software used to prepare material for publication: $\operatorname{Win} G X$ (Farrugia, 2012).

2-Amino-3-hydroxypyridin-1-ium 6-methyl-2,2,4-trioxo-2H,4H-1,2,3-oxathiazin-3-ide

Crystal data

$\mathrm{C}_{5} \mathrm{H}_{7} \mathrm{~N}_{2} \mathrm{O}^{+} \cdot \mathrm{C}_{4} \mathrm{H}_{4} \mathrm{NO}_{4} \mathrm{~S}^{-}$

$M_{r}=273.27$

Triclinic, $P \overline{1}$

$a=7.1676(5) \AA$

$b=9.1175(7) \AA$

$c=10.1554(8) \AA$

$\alpha=66.174(6)^{\circ}$

$\beta=80.225(6)^{\circ}$

$\gamma=71.803(6)^{\circ}$

$V=576.01(8) \AA^{3}$

Data collection

STOE IPDS 2 diffractometer

Radiation source: sealed X-ray tube, $12 \times 0.4$ $\mathrm{mm}$ long-fine focus

Detector resolution: 6.67 pixels $\mathrm{mm}^{-1}$

rotation method scans

Absorption correction: integration

(X-RED32; Stoe \& Cie, 2002)

$T_{\text {min }}=0.855, T_{\text {max }}=0.953$

Refinement

Refinement on $F^{2}$

Least-squares matrix: full

$R\left[F^{2}>2 \sigma\left(F^{2}\right)\right]=0.040$

$w R\left(F^{2}\right)=0.114$

$S=1.08$

2263 reflections
$Z=2$

$F(000)=284$

$D_{\mathrm{x}}=1.576 \mathrm{Mg} \mathrm{m}^{-3}$

Mo $K \alpha$ radiation, $\lambda=0.71073 \AA$

Cell parameters from 10423 reflections

$\theta=3.0-31.5^{\circ}$

$\mu=0.30 \mathrm{~mm}^{-1}$

$T=296 \mathrm{~K}$

Prism, red

$0.57 \times 0.42 \times 0.21 \mathrm{~mm}$

5116 measured reflections

2263 independent reflections

1951 reflections with $I>2 \sigma(I)$

$R_{\text {int }}=0.031$

$\theta_{\text {max }}=26.0^{\circ}, \theta_{\min }=3.0^{\circ}$

$h=-8 \rightarrow 8$

$k=-10 \rightarrow 11$

$l=-12 \rightarrow 12$

179 parameters

0 restraints

Primary atom site location: structure-invariant direct methods

Secondary atom site location: difference Fourier map 
Hydrogen site location: mixed

$\mathrm{H}$ atoms treated by a mixture of independent and constrained refinement

$$
\begin{aligned}
& w=1 /\left[\sigma^{2}\left(F_{\mathrm{o}}^{2}\right)+(0.0615 P)^{2}+0.1608 P\right] \\
& \text { where } P=\left(F_{\mathrm{o}}^{2}+2 F_{\mathrm{c}}^{2}\right) / 3 \\
& (\Delta / \sigma)_{\max }=0.001 \\
& \Delta \rho_{\max }=0.34 \mathrm{e} \AA^{-3} \\
& \Delta \rho_{\min }=-0.26 \mathrm{e} \AA^{-3}
\end{aligned}
$$

Special details

Geometry. All esds (except the esd in the dihedral angle between two 1.s. planes) are estimated using the full covariance

\begin{tabular}{|c|c|c|c|c|}
\hline & $x$ & $y$ & $z$ & $U_{\text {iso }} * / U_{\text {eq }}$ \\
\hline $\mathrm{S} 1$ & $1.03944(7)$ & $0.17969(7)$ & $0.69020(6)$ & $0.04075(18)$ \\
\hline O1 & $0.9160(2)$ & 0.10735 (19) & $0.62259(17)$ & $0.0478(4)$ \\
\hline $\mathrm{O} 3$ & $1.0909(3)$ & $0.3136(2)$ & $0.57109(18)$ & $0.0601(5)$ \\
\hline O5 & $0.1101(3)$ & $0.5582(2)$ & $0.70493(17)$ & $0.0553(4)$ \\
\hline $\mathrm{O} 4$ & $0.5989(2)$ & $0.3846(3)$ & $0.8548(2)$ & $0.0666(5)$ \\
\hline N2 & -0.0570 & $0.7569(2)$ & $0.9560(2)$ & $0.0438(4)$ \\
\hline $\mathrm{O} 2$ & $1.1970(2)$ & $0.0414(2)$ & $0.7566(2)$ & $0.0659(5)$ \\
\hline N3 & 0.2510 & 0.6079 & $0.9066(2)$ & $0.0455(4)$ \\
\hline N1 & $0.8981(2)$ & $0.2371(2)$ & 0.80563 (19) & $0.0451(4)$ \\
\hline $\mathrm{C} 5$ & 0.0626 & $0.6702(2)$ & $0.8807(2)$ & $0.0371(4)$ \\
\hline $\mathrm{C} 3$ & 0.7205 & $0.1932(3)$ & $0.5985(2)$ & $0.0407(4)$ \\
\hline $\mathrm{C} 1$ & 0.7049 & 0.3113 & $0.7784(2)$ & $0.0441(5)$ \\
\hline C6 & $-0.0206(3)$ & 0.6490 & $0.7748(2)$ & $0.0435(5)$ \\
\hline $\mathrm{C} 2$ & $0.6223(3)$ & $0.2932(3)$ & $0.6663(2)$ & $0.0461(5)$ \\
\hline $\mathrm{H} 2 \mathrm{~A}$ & 0.494302 & 0.354995 & 0.641245 & $0.055^{*}$ \\
\hline C7 & $-0.2159(4)$ & $0.7151(3)$ & $0.7551(2)$ & $0.0565(6)$ \\
\hline $\mathrm{H} 7$ & -0.271871 & 0.700166 & 0.686842 & $0.068^{*}$ \\
\hline $\mathrm{C} 9$ & $-0.2527(3)$ & $0.8245(3)$ & $0.9352(3)$ & $0.0564(6)$ \\
\hline H9 & -0.330076 & 0.884168 & 0.990109 & $0.068^{*}$ \\
\hline $\mathrm{C} 8$ & $-0.3342(3)$ & $0.8056(4)$ & $0.8363(3)$ & $0.0636(7)$ \\
\hline H8 & -0.467976 & 0.852033 & 0.821546 & $0.076^{*}$ \\
\hline $\mathrm{C} 4$ & $0.6425(4)$ & $0.1525(4)$ & $0.4958(3)$ & $0.0565(6)$ \\
\hline $\mathrm{H} 4 \mathrm{~A}$ & 0.745607 & 0.076688 & 0.462632 & $0.085^{*}$ \\
\hline H4B & 0.537932 & 0.101580 & 0.542857 & $0.085^{*}$ \\
\hline $\mathrm{H} 4 \mathrm{C}$ & 0.593203 & 0.252581 & 0.415180 & $0.085^{*}$ \\
\hline $\mathrm{H} 3 \mathrm{~A}$ & $0.289(4)$ & $0.614(3)$ & $0.978(3)$ & $0.054(7)^{*}$ \\
\hline H3B & $0.320(4)$ & $0.547(3)$ & $0.867(3)$ & $0.053(7)^{*}$ \\
\hline H5 & $0.048(6)$ & $0.554(5)$ & $0.644(4)$ & $0.105(12)^{*}$ \\
\hline $\mathrm{H} 2$ & $-0.014(4)$ & $0.775(4)$ & $1.024(3)$ & $0.066(8)^{*}$ \\
\hline
\end{tabular}
matrix. The cell esds are taken into account individually in the estimation of esds in distances, angles and torsion angles; correlations between esds in cell parameters are only used when they are defined by crystal symmetry. An approximate (isotropic) treatment of cell esds is used for estimating esds involving l.s. planes.

Fractional atomic coordinates and isotropic or equivalent isotropic displacement parameters $\left(\AA^{2}\right)$

Atomic displacement parameters $\left(\AA^{2}\right)$

\begin{tabular}{lllllll}
\hline & $U^{11}$ & $U^{22}$ & $U^{33}$ & $U^{12}$ & $U^{13}$ & $U^{23}$ \\
\hline $\mathrm{S} 1$ & $0.0320(3)$ & $0.0515(3)$ & $0.0452(3)$ & $-0.0048(2)$ & $-0.00460(19)$ & $-0.0282(2)$
\end{tabular}




\begin{tabular}{lllllll} 
O1 & $0.0402(8)$ & $0.0550(9)$ & $0.0611(9)$ & $-0.0052(7)$ & $-0.0055(7)$ & $-0.0393(8)$ \\
O3 & $0.0616(10)$ & $0.0769(12)$ & $0.0537(9)$ & $-0.0322(9)$ & $0.0023(8)$ & $-0.0281(9)$ \\
O5 & $0.0630(10)$ & $0.0730(11)$ & $0.0459(9)$ & $-0.0222(9)$ & $-0.0056(8)$ & $-0.0342(8)$ \\
O4 & $0.0420(8)$ & $0.1016(14)$ & $0.0750(11)$ & $0.0038(9)$ & $-0.0074(8)$ & $-0.0674(11)$ \\
N2 & $0.0387(9)$ & $0.0502(10)$ & $0.0425(9)$ & $-0.0055(8)$ & $-0.0056(7)$ & $-0.0209(8)$ \\
O2 & $0.0425(9)$ & $0.0720(12)$ & $0.0812(12)$ & $0.0127(8)$ & $-0.0196(8)$ & $-0.0414(10)$ \\
N3 & $0.0394(9)$ & $0.0572(11)$ & $0.0463(10)$ & $-0.0011(8)$ & $-0.0112(8)$ & $-0.0312(9)$ \\
N1 & $0.0343(8)$ & $0.0645(11)$ & $0.0460(9)$ & $-0.0050(8)$ & $-0.0062(7)$ & $-0.0349(9)$ \\
C5 & $0.0383(10)$ & $0.0395(10)$ & $0.0325(9)$ & $-0.0097(8)$ & $-0.0047(7)$ & $-0.0118(8)$ \\
C3 & $0.0386(10)$ & $0.0495(11)$ & $0.0404(10)$ & $-0.0119(9)$ & $-0.0054(8)$ & $-0.0218(9)$ \\
C1 & $0.0363(10)$ & $0.0569(12)$ & $0.0459(11)$ & $-0.0052(9)$ & $-0.0032(8)$ & $-0.0311(10)$ \\
C6 & $0.0492(11)$ & $0.0508(12)$ & $0.0328(9)$ & $-0.0200(9)$ & $-0.0067(8)$ & $-0.0113(9)$ \\
C2 & $0.0338(10)$ & $0.0596(13)$ & $0.0481(11)$ & $-0.0017(9)$ & $-0.0102(9)$ & $-0.0283(10)$ \\
C7 & $0.0522(13)$ & $0.0729(16)$ & $0.0446(12)$ & $-0.0244(12)$ & $-0.0171(10)$ & $-0.0109(12)$ \\
C9 & $0.0389(11)$ & $0.0605(14)$ & $0.0590(14)$ & $-0.0012(10)$ & $-0.0020(10)$ & $-0.0209(12)$ \\
C8 & $0.0370(12)$ & $0.0758(17)$ & $0.0612(15)$ & $-0.0102(11)$ & $-0.0122(11)$ & $-0.0089(13)$ \\
C4 & $0.0589(14)$ & $0.0759(16)$ & $0.0524(13)$ & $-0.0233(12)$ & $-0.0076(11)$ & $-0.0361(12)$ \\
& & & & & & \\
\hline
\end{tabular}

Geometric parameters (A, $\stackrel{\circ}{)}$

\begin{tabular}{llll}
\hline $\mathrm{S} 1-\mathrm{O} 2$ & $1.4149(17)$ & $\mathrm{C} 5-\mathrm{C} 6$ & $1.417(3)$ \\
$\mathrm{S} 1-\mathrm{O} 3$ & $1.4235(18)$ & $\mathrm{C} 3-\mathrm{C} 2$ & $1.320(3)$ \\
$\mathrm{S} 1-\mathrm{N} 1$ & $1.5605(17)$ & $\mathrm{C} 3-\mathrm{C} 4$ & $1.476(3)$ \\
$\mathrm{S} 1-\mathrm{O} 1$ & $1.6204(15)$ & $\mathrm{C} 1-\mathrm{C} 2$ & $1.454(3)$ \\
$\mathrm{O} 1-\mathrm{C} 3$ & $1.383(2)$ & $\mathrm{C} 6-\mathrm{C} 7$ & $1.354(3)$ \\
$\mathrm{O} 5-\mathrm{C} 6$ & $1.353(3)$ & $\mathrm{C} 2-\mathrm{H} 2 \mathrm{~A}$ & 1.9300 \\
$\mathrm{O} 5-\mathrm{H} 5$ & $0.84(4)$ & $\mathrm{C} 7-\mathrm{C} 8$ & 0.9300 \\
$\mathrm{O} 4-\mathrm{C} 1$ & $1.236(3)$ & $\mathrm{C} 7-\mathrm{H} 7$ & $1.336(4)$ \\
$\mathrm{N} 2-\mathrm{C} 5$ & $1.336(3)$ & $\mathrm{C} 9-\mathrm{C} 8$ & 0.9300 \\
$\mathrm{~N} 2-\mathrm{C} 9$ & $1.359(3)$ & $\mathrm{C} 9-\mathrm{H} 9$ & 0.9300 \\
$\mathrm{~N} 2-\mathrm{H} 2$ & $0.90(3)$ & $\mathrm{C} 8-\mathrm{H} 8$ & 0.9600 \\
$\mathrm{~N} 3-\mathrm{C} 5$ & $1.317(3)$ & $\mathrm{C} 4-\mathrm{H} 4 \mathrm{~A}$ & 0.9600 \\
$\mathrm{~N} 3-\mathrm{H} 3 \mathrm{~A}$ & $0.85(3)$ & $\mathrm{C} 4-\mathrm{H} 4 \mathrm{~B}$ & 0.9600 \\
$\mathrm{~N} 3-\mathrm{H} 3 \mathrm{~B}$ & $0.81(3)$ & $\mathrm{C} 4-\mathrm{H} 4 \mathrm{C}$ & \\
$\mathrm{N} 1-\mathrm{C} 1$ & $1.359(3)$ & & $119.43(18)$ \\
& & & $126.8(2)$ \\
$\mathrm{O} 2-\mathrm{S} 1-\mathrm{O} 3$ & $116.38(12)$ & $\mathrm{N} 1-\mathrm{C} 1-\mathrm{C} 2$ & $113.93(18)$ \\
$\mathrm{O} 2-\mathrm{S} 1-\mathrm{N} 1$ & $110.55(11)$ & $\mathrm{O} 5-\mathrm{C} 6-\mathrm{C} 7$ & $119.2(2)$ \\
$\mathrm{O} 3-\mathrm{S} 1-\mathrm{N} 1$ & $113.19(11)$ & $\mathrm{O} 5-\mathrm{C} 6-\mathrm{C} 5$ & $123.12(19)$ \\
$\mathrm{O} 2-\mathrm{S} 1-\mathrm{O} 1$ & $104.59(10)$ & $\mathrm{C} 7-\mathrm{C} 6-\mathrm{C} 5$ & 118.4 \\
$\mathrm{O} 3-\mathrm{S} 1-\mathrm{O} 1$ & $104.86(9)$ & $\mathrm{C} 3-\mathrm{C} 2-\mathrm{C} 1$ & 118.4 \\
$\mathrm{~N} 1-\mathrm{S} 1-\mathrm{O} 1$ & $106.18(9)$ & $\mathrm{C} 3-\mathrm{C} 2-\mathrm{H} 2 \mathrm{~A}$ & $120.7(2)$ \\
$\mathrm{C} 3-\mathrm{O} 1-\mathrm{S} 1$ & $116.85(13)$ & $\mathrm{C} 1-\mathrm{C} 2-\mathrm{H} 2 \mathrm{~A}$ & 119.7 \\
$\mathrm{C} 6-\mathrm{O} 5-\mathrm{H} 5$ & $107(3)$ & $\mathrm{C} 6-\mathrm{C} 7-\mathrm{C} 8$ & 119.7 \\
$\mathrm{C} 5-\mathrm{N} 2-\mathrm{C} 9$ & $123.0(2)$ & $\mathrm{C} 6-\mathrm{C} 7-\mathrm{H} 7$ & $120.2(2)$ \\
$\mathrm{C} 5-\mathrm{N} 2-\mathrm{H} 2$ & $122.3(18)$ & $\mathrm{C} 8-\mathrm{C} 7-\mathrm{H} 7$ & 119.9 \\
$\mathrm{C} 9-\mathrm{N} 2-\mathrm{H} 2$ & $114.6(18)$ & $\mathrm{C} 8-\mathrm{C} 9-\mathrm{N} 2-\mathrm{C} 2$ & \\
$\mathrm{C} 5-\mathrm{N} 3-\mathrm{H} 3 \mathrm{~A}$ & $117.7(18)$ & &
\end{tabular}




$\begin{array}{llll}\mathrm{C} 5-\mathrm{N} 3-\mathrm{H} 3 \mathrm{~B} & 118.7(19) & \mathrm{N} 2-\mathrm{C} 9-\mathrm{H} 9 & 119.9 \\ \mathrm{H} 3 \mathrm{~A}-\mathrm{N} 3-\mathrm{H} 3 \mathrm{~B} & 122(3) & \mathrm{C} 9-\mathrm{C} 8-\mathrm{C} 7 & 119.2(2) \\ \mathrm{C} 1-\mathrm{N} 1-\mathrm{S} 1 & 118.98(14) & \mathrm{C} 9-\mathrm{C} 8-\mathrm{H} 8 & 120.4 \\ \mathrm{~N} 3-\mathrm{C} 5-\mathrm{N} 2 & 119.84(18) & \mathrm{C} 7-\mathrm{C} 8-\mathrm{H} 8 & 120.4 \\ \mathrm{~N} 3-\mathrm{C} 5-\mathrm{C} 6 & 122.40(19) & \mathrm{C} 3-\mathrm{C} 4-\mathrm{H} 4 \mathrm{~A} & 109.5 \\ \mathrm{~N} 2-\mathrm{C} 5-\mathrm{C} 6 & 117.77(18) & \mathrm{C} 3-\mathrm{C} 4-\mathrm{H} 4 \mathrm{~B} & 109.5 \\ \mathrm{C} 2-\mathrm{C} 3-\mathrm{O} 1 & 121.46(18) & \mathrm{H} 4 \mathrm{~A}-\mathrm{C} 4-\mathrm{H} 4 \mathrm{~B} & 109.5 \\ \mathrm{C} 2-\mathrm{C} 3-\mathrm{C} 4 & 126.9(2) & \mathrm{C} 3-\mathrm{C} 4-\mathrm{H} 4 \mathrm{C} & 109.5 \\ \mathrm{O} 1-\mathrm{C} 3-\mathrm{C} 4 & 111.62(18) & \mathrm{H} 4 \mathrm{~A}-\mathrm{C} 4-\mathrm{H} 4 \mathrm{C} & 109.5 \\ \mathrm{O} 4-\mathrm{C} 1-\mathrm{N} 1 & 119.67(18) & \mathrm{H} 4 \mathrm{~B}-\mathrm{C} 4-\mathrm{H} 4 \mathrm{C} & \\ \mathrm{O} 4-\mathrm{C} 1-\mathrm{C} 2 & 120.74(19) & & -179.25(18) \\ & & & 179.3(2) \\ \mathrm{O} 2-\mathrm{S} 1-\mathrm{O} 1-\mathrm{C} 3 & 156.84(16) & \mathrm{N} 2-\mathrm{C} 5-\mathrm{C} 6-\mathrm{O} 5 & -0.6(3) \\ \mathrm{O} 3-\mathrm{S} 1-\mathrm{O} 1-\mathrm{C} 3 & -80.20(17) & \mathrm{N} 3-\mathrm{C} 5-\mathrm{C} 6-\mathrm{C} 7 & -4.9(4) \\ \mathrm{N} 1-\mathrm{S} 1-\mathrm{O} 1-\mathrm{C} 3 & 39.88(17) & \mathrm{N} 2-\mathrm{C} 5-\mathrm{C} 6-\mathrm{C} 7 & 172.6(2) \\ \mathrm{O} 2-\mathrm{S} 1-\mathrm{N} 1-\mathrm{C} 1 & -151.14(19) & \mathrm{O} 1-\mathrm{C} 3-\mathrm{C} 2-\mathrm{C} 1 & -168.2(2) \\ \mathrm{O} 3-\mathrm{S} 1-\mathrm{N} 1-\mathrm{C} 1 & 76.3(2) & \mathrm{C} 4-\mathrm{C} 3-\mathrm{C} 2-\mathrm{C} 1 & 7.4(4) \\ \mathrm{O} 1-\mathrm{S} 1-\mathrm{N} 1-\mathrm{C} 1 & -38.2(2) & \mathrm{O} 4-\mathrm{C} 1-\mathrm{C} 2-\mathrm{C} 3 & 179.5(2) \\ \mathrm{C} 9-\mathrm{N} 2-\mathrm{C} 5-\mathrm{N} 3 & -179.9(2) & \mathrm{N} 1-\mathrm{C} 1-\mathrm{C} 2-\mathrm{C} 3 & 1.0(4) \\ \mathrm{C} 9-\mathrm{N} 2-\mathrm{C} 5-\mathrm{C} 6 & 0.0(3) & \mathrm{O} 5-\mathrm{C} 6-\mathrm{C} 7-\mathrm{C} 8 & 0.2(4) \\ \mathrm{S} 1-\mathrm{O} 1-\mathrm{C} 3-\mathrm{C} 2 & -20.9(3) & \mathrm{C} 5-\mathrm{C} 6-\mathrm{C} 7-\mathrm{C} 8 & 0.2(4) \\ \mathrm{S} 1-\mathrm{O} 1-\mathrm{C} 3-\mathrm{C} 4 & 161.22(16) & \mathrm{C} 5-\mathrm{N} 2-\mathrm{C} 9-\mathrm{C} 8 & -0.8(4) \\ \mathrm{S} 1-\mathrm{N} 1-\mathrm{C} 1-\mathrm{O} 4 & -166.63(19) & \mathrm{N} 2-\mathrm{C} 9-\mathrm{C} 8-\mathrm{C} 7 & \\ \mathrm{~S} 1-\mathrm{N} 1-\mathrm{C} 1-\mathrm{C} 2 & 17.7(3) & \mathrm{C} 6-\mathrm{C} 7-\mathrm{C} 8-\mathrm{C} 9 & \\ \mathrm{~N} 3-\mathrm{C} 5-\mathrm{C} 6-\mathrm{O} 5 & 0.7(3) & & \end{array}$

Hydrogen-bond geometry $\left(\AA,{ }^{\circ}\right)$

\begin{tabular}{lllll}
\hline$D-\mathrm{H} \cdots A$ & $D-\mathrm{H}$ & $\mathrm{H} \cdots A$ & $D \cdots A$ & $D-\mathrm{H} \cdots A$ \\
\hline $\mathrm{N} 3-\mathrm{H} 3 B \cdots \mathrm{O} 4$ & $0.81(3)$ & $2.10(3)$ & $2.808(3)$ & $145(2)$ \\
$\mathrm{N} 3-\mathrm{H} 3 A \cdots \mathrm{O} 4{ }^{\mathrm{i}}$ & $0.85(3)$ & $2.00(3)$ & $2.846(3)$ & $175(3)$ \\
$\mathrm{N} 2-\mathrm{H} 2 \cdots \mathrm{N} 1^{\mathrm{i}}$ & $0.90(3)$ & $1.99(3)$ & $2.871(3)$ & $168(3)$ \\
$\mathrm{O} 5-\mathrm{H} 5 \cdots \mathrm{O} 3^{i i}$ & $0.84(4)$ & $2.50(4)$ & $3.090(2)$ & $128(3)$ \\
$\mathrm{O} 5-\mathrm{H} 5 \cdots 3^{i i i}$ & $0.84(4)$ & $2.25(4)$ & $2.995(2)$ & $147(3)$ \\
$\mathrm{C} 8-\mathrm{H} 8 \cdots \mathrm{O} 2^{\text {iv }}$ & 0.93 & 2.49 & $3.402(3)$ & 166 \\
\hline
\end{tabular}

Symmetry codes: (i) $-x+1,-y+1,-z+2$; (ii) $x-1, y, z$; (iii) $-x+1,-y+1,-z+1$; (iv) $x-2, y+1, z$. 